\title{
Radiomic Analysis Reveals Prognostic Information in T1-Weighted Baseline Magnetic Resonance Imaging in Patients With Glioblastoma
}

\author{
Michael Ingrisch, PhD, * Moritz Jörg Schneider, MSc, * Dominik Nörenberg, MD, † \\ Giovanna Negrao de Figueiredo, MD, † Klaus Maier-Hein, PhD, $\neq$ Bogdana Suchorska, MD, $\S$ \\ Ulrich Schüller, MD,// Nathalie Albert, MD,\# Hartmut Brückmann, MD, ** Maximilian Reiser, MD, $†$ \\ Jörg-Christian Tonn, MD, $\S$ and Birgit Ertl-Wagner, MD†
}

Objectives: The aim of this study was to investigate whether radiomic analysis with random survival forests (RSFs) can predict overall survival from T1weighted contrast-enhanced baseline magnetic resonance imaging (MRI) scans in a cohort of glioblastoma multiforme (GBM) patients with uniform treatment. Materials and Methods: This retrospective study was approved by the institutional review board and informed consent was waived. The MRI scans from 66 patients with newly diagnosed GBM from a previous prospective study were analyzed. Tumors were segmented manually on contrast-enhanced 3-dimensional T1-weighted images. Using these segmentations, $P=208$ quantitative image features characterizing tumor shape, signal intensity, and texture were calculated in an automated fashion. On this data set, an RSF was trained using 10-fold cross validation to establish a link between image features and overall survival, and the individual risk for each patient was predicted. The mean concordance index was assessed as a measure of prediction accuracy. Association of individual risk with overall survival was assessed using Kaplan-Meier analysis and a univariate proportional hazards model.

Results: Mean overall survival was 14 months (range, $0.8-85$ months). Mean concordance index of the 10-fold cross-validated RSF was 0.67. Kaplan-Meier analysis clearly distinguished 2 patient groups with high and low predicted individual risk $\left(P=5.5 \times 10^{-5}\right)$. Low predicted individual mortality was found to be a favorable prognostic factor for overall survival in a univariate Cox proportional hazards model (hazards ratio, 1.038; 95\% confidence interval, 1.015-1.062; $P=0.0059$ ). Conclusions: This study demonstrates that baseline MRI in GBM patients contains prognostic information, which can be accessed by radiomic analysis using RSFs.

Key Words: radiomics, glioblastoma, survival, random survival forest

(Invest Radiol 2017;52: 360-366)

Received November 9, 2016; accepted after revision November 30, 2016

From the *Josef Lissner Laboratory for Biomedical Imaging, and †nstitute for Clinical Radiology, Ludwig-Maximilians University Hospital Munich, Munich; \$Medical Image Computing, German Cancer Research Center (DKFZ), Heidelberg; §Department of Neurosurgery, and $\|$ Center for Neuropathology and Prion Research, Ludwig-Maximilian University Hospital Munich, Munich, Germany; 『Research Institute Children's Cancer Center, Hamburg; Departments of \#Nuclear Medicine, and **Neuroradiology, Ludwig-Maximilian University Hospital, Munich, Germany.

Conflicts of interest and sources of funding: This publication was funded by LudwigMaximilian University Munich's Institutional Strategy LMU excellent within the framework of the German Excellence Initiative. J.C.T. received honoraria and travel grants from BrainLab and Siemens. All other authors have no conflicts of interest to declare.

Supplemental digital contents are available for this article. Direct URL citations appear in the printed text and are provided in the HTML and PDF versions of this article on the journal's Web site (www.investigativeradiology.com)

Correspondence to: Michael Ingrisch, PhD, Josef Lissner Laboratory for Biomedical Imaging, Institute for Clinical Radiology, Ludwig-Maximilian University Hospital, Marchioninistrasse 15, 81377 Munich, Germany. E-mail: michael. ingrisch@med.lmu.de.

Copyright (c) 2017 Wolters Kluwer Health, Inc. All rights reserved.

ISSN: 0020-9996/17/5206-0360

DOI: $10.1097 /$ RLI.0000000000000349
G lioblastoma multiforme (GBM) is a malignant primary brain tumor with a poor prognosis and median survival times between 11 and 15 months. ${ }^{1-6}$ In clinical routine, GBMs are usually diagnosed and followed-up with MRI. Extracting in-depth prognostic data and novel imaging biomarkers from these routinely acquired image data sets would have a high relevance for personalized and precise therapeutic decision making and potentially for the development and implementation of new treatment approaches. ${ }^{7,8}$

Recently, radiomic approaches have emerged as promising tools to enhance information attainable from imaging by means of automated high-throughput analysis combined with machine learning. ${ }^{7,9,10}$ This relies on converting radiological images into a large number of quantitative image features that characterize numerous aspects of the image. The resulting parameter space is then subjected to data mining methods to extract meaningful information. Recently, this was used, for example, to classify prostate cancer ${ }^{11}$ or breast cancer ${ }^{12}$ based on MRI scans or to predict survival ${ }^{7,13}$ or distant metastases ${ }^{14}$ in non-small cell lung cancer based on CT data.

A radiomic analysis generates a high-dimensional parameter space, where the number of parameters typically exceeds the number of samples by far. This inherent quality of radiomic data sets poses a significant challenge for statistical data analysis: the large number of image features renders classical regression approaches unfeasible and potentially strong correlations between similar image features complicate the selection and identification of strong predictive variables. Such high-dimensional problems may be addressed with machine learning methods such as random forests (RFs) originally proposed for classification problems. ${ }^{15}$ Random forests have recently been extended to deal with right-censored survival data ${ }^{16,17}$; the outcome variable of such random survival forests (RSFs) is an individual measure of patient risk as a predictor of overall survival. ${ }^{18}$ Unlike conventional survival models, RSFs are nonparametric, do not rely on restrictive assumptions such as proportional hazards, and can easily incorporate variable interactions. Random survival forests can also be used to identify prognostic variables and have been used to identify risk factors in patients with systolic heart failure ${ }^{19}$ and to identify variables associated with recurrence of hepatocellular carcinoma. ${ }^{20}$

The purpose of this retrospective single center study was therefore to investigate whether a radiomic analysis with RSF can extract prognostic information from T1-weighted contrast-enhanced (CE) baseline MRI scans in a cohort of GBM patients with uniform treatment.

\section{MATERIALS AND METHODS}

\section{Patients and MRI}

This retrospective study was approved by the institutional review board of the Ludwig-Maximilian University, Munich, Germany (241-15). Patients were included from a previous prospective study (NCT01089868 $8^{21}$ ), for which written informed consent had been 

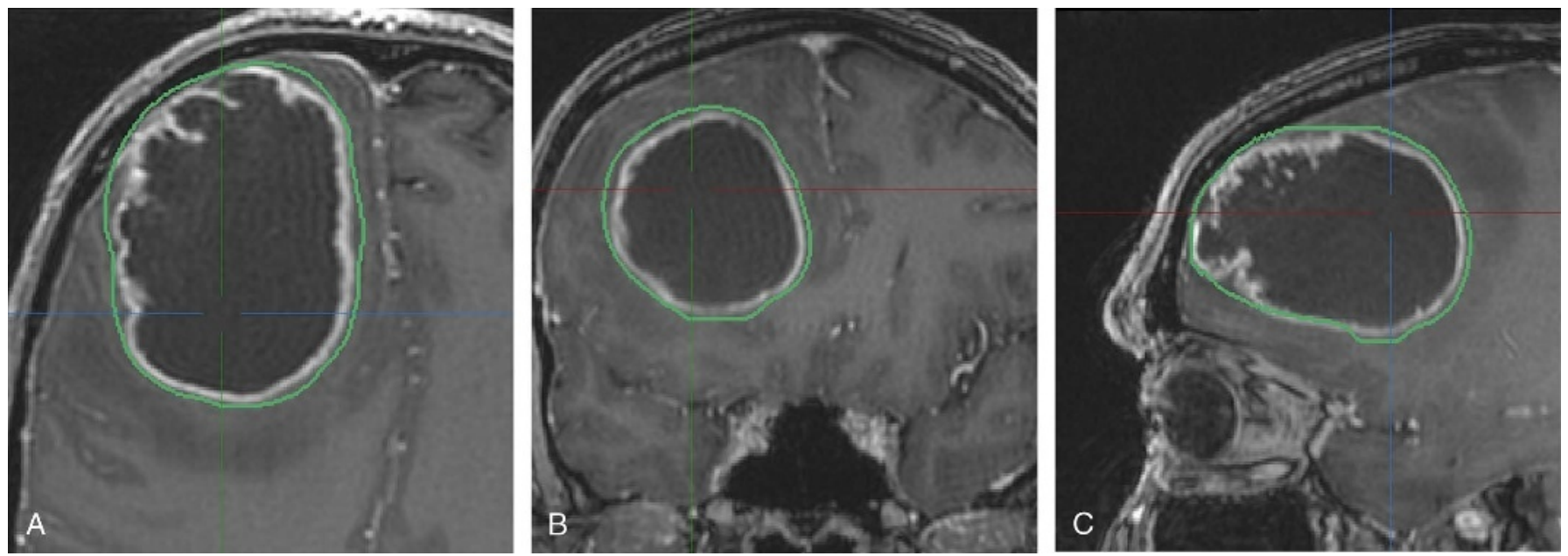

FIGURE 1. GBM segmentation of contrast-enhancing tumor volume on 3 T CE T1-weighted images of a 58-year-old GBM patient with an overall survival of 79 months. After initial imaging, the patient received microsurgery and completed RCX. Axial (A), coronal (B), and sagittal slice (C), along with the segmentation that was used for feature calculation. Figure 1 can be viewed online in color at www.investigativeradiology.com.

obtained. This initial prospective trial investigated the prognostic value of positron emission tomography on survival of GBM patients with standardized therapy, whereas in the present manuscript, we analyze the prognostic value of baseline MRI data. Retrospective informed consent for the additional data analysis was waived by the institutional review board. Inclusion range was February 2007 to January 2010, the date of last follow-up was February 28, 2014, and the study end point was overall survival. Inclusion criteria were as follows: histologically proven GBM by stereotactic biopsy or by open tumor resection according to the World Health Organization classification, ${ }^{1}$ no prior treatment such as radiotherapy or chemotherapy, no history of surgery, baseline MRI performed in our institution, and availability of 3-dimensional (3D) CE T1-weighted baseline MRI scans.
Magnetic resonance imaging scans were acquired either on a 1.5 T system (Magnetom Symphony; Siemens Healthcare, Erlangen, Germany) or on a 3 T system (Signa HDx; GE Healthcare, Milwaukee, WI). Imaging parameters varied over time and magnet type (in-plane resolution, $0.5-1 \mathrm{~mm}$ per pixel; slice thickness, $1.2-1.6 \mathrm{~mm}$; echo time, 3.2-4.8 milliseconds; repetition time, 6-12 milliseconds; flip angle, 15 degrees).

\section{Image Analysis and Feature Extraction}

All MRI scans were reviewed independently in a randomized fashion and blinded to the clinical data, only 3D CE T1-weighted MRI scans were used for further analysis. Tumor segmentation was performed using the Medical Image Interaction Toolkit. ${ }^{22}$ Contrast-enhancing

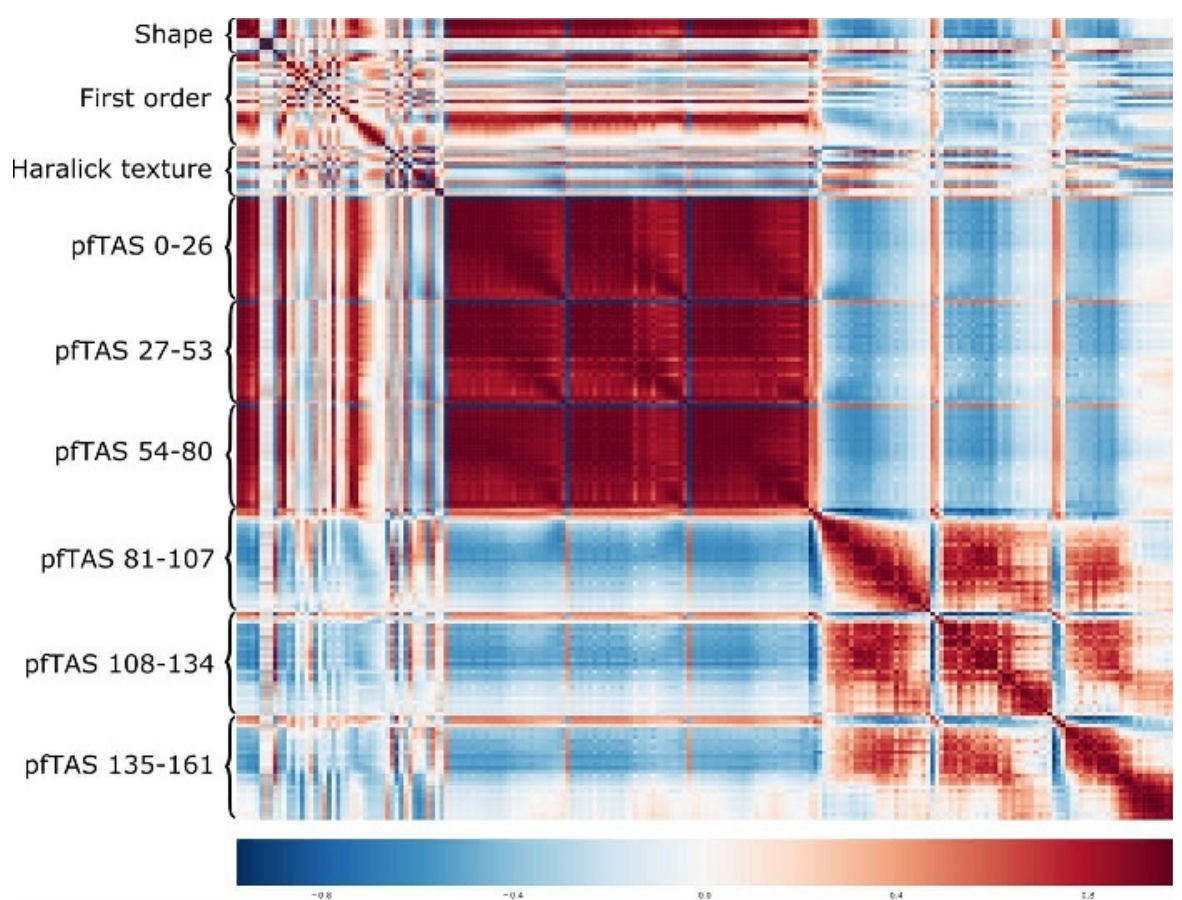

FIGURE 2. Correlation matrix of the 208 image features, showing blocks with high positive (red) and negative (blue) correlations between several groups of features. Figure 2 can be viewed online in color at www.investigativeradiology.com. 
tumor regions were outlined manually on orthogonal slices of CE T1weighted images, after the contrast-enhancing tumor outline. Using this contour information, the tumor surface was interpolated using the $3 \mathrm{D}$ interpolation feature of Medical Image Interaction Toolkit. Contours were added until the interpolated surface closely matched the tumor outline on all slices. The resulting 3D tumor segmentation included contrast-enhancing tumor tissue and central necrosis, these habitats were not further discriminated. In patients with more than 1 lesion, each lesion was contoured individually.

Before further analysis, images and segmentations were resampled to $0.5 \times 0.5 \times 0.5 \mathrm{~mm}^{3}$ voxel size. Images were normalized and contrast stretched to values from the minimum to the 98th percentile. To turn images into mineable data, a set of $P=208$ quantitative image features was extracted in a fully automated fashion from each segmentation on CE T1-weighted images. The shape of the segmentations was quantified by 9 features reflecting the total volume, surface area, and sphericity among others. ${ }^{7}$ Twenty-four features describe first-order intensity statistics such as median signal intensity or standard deviation and were derived from histograms of the signal intensity. ${ }^{7}$ The remaining features characterized tumor texture, conveying information about the spatial arrangement of the signal intensity distribution. These include $n=13$ Haralick features ${ }^{23,24}$ and $n=162$ parameter-free threshold adjacency statistics (pfTAS). ${ }^{24-26}$ The pfTAS features are derived from images by thresholding the tumor volume and counting the number of adjacent black or white pixels for each pixel. In patients with more than 1 lesion, the individual segmentations were combined and image features were calculated cumulatively for all lesions.

Details about the individual features are described in the Supplementary Appendix, Supplemental Digital Content 1, http://links.lww. com/RLI/A307. Feature extraction routines were implemented in Python as nipype nodes, ${ }^{27}$ thus allowing for parallel computation in a fully automated feature extraction pipeline. Calculations were performed on a Linux workstation (Intel i7 CPU, 32 GB memory).

\section{Statistical Analysis}

Statistical modeling was performed in $\mathrm{R}$ (version 3.3.1,2016 ${ }^{28}$ ). Univariate Cox proportional hazards models were used to assess the effects of clinical variables (age, sex, mode of surgery, Karnofsky performance score [KPS]) on overall survival; $P$ values of the resulting univariate models were adjusted for multiple testing using the Holm correction. ${ }^{29}$

An $\mathrm{RSF}^{17}$ was used to build a prognostic model for overall survival using the $\mathrm{R}$ package randomForestRSC. ${ }^{30}$ All $P=208$ image

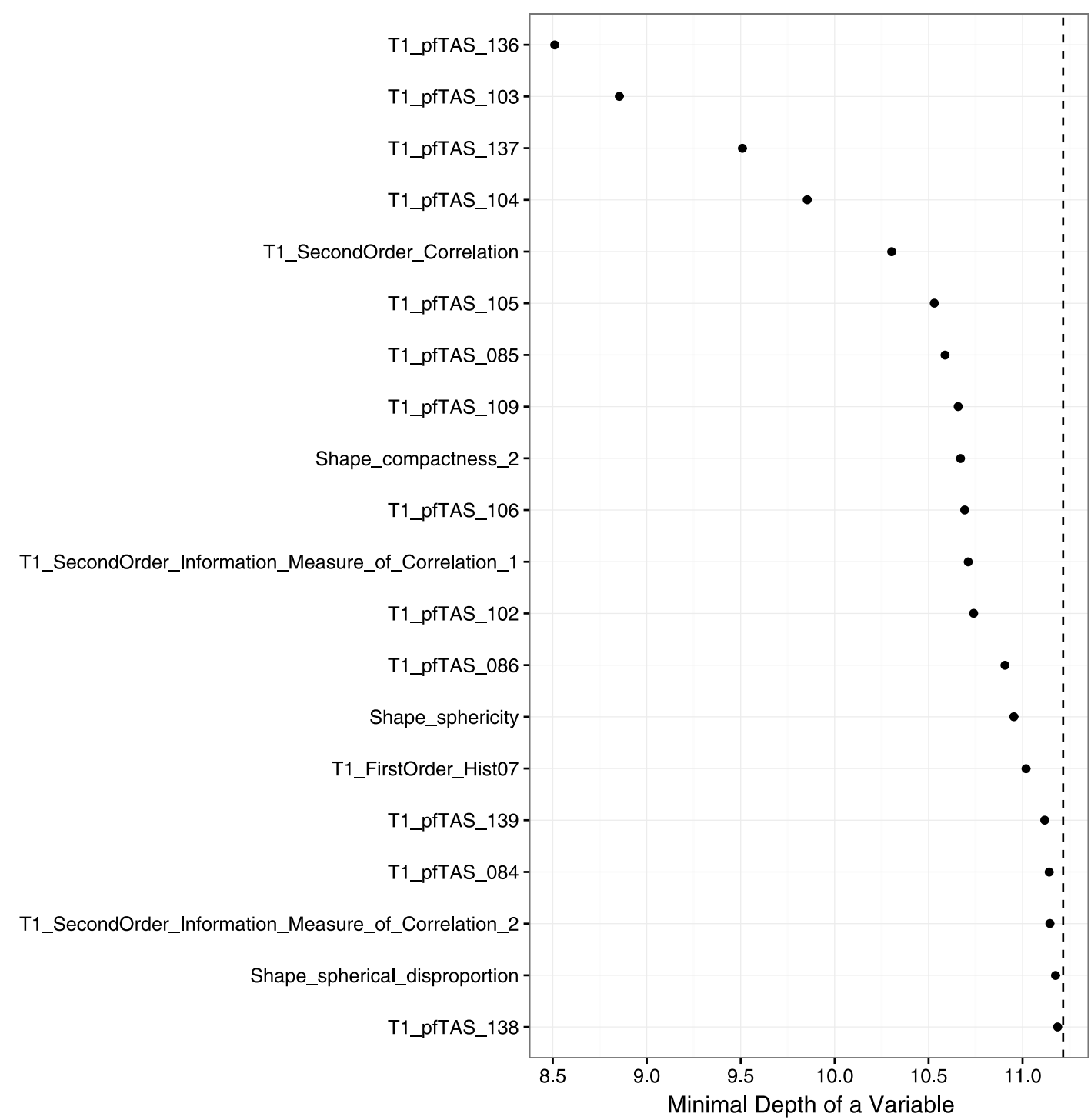

FIGURE 3. Variable importance of prognostic features, as determined by minimal depth variable selection. 
features were used for the analysis. Dimensionality reduction was performed by "minimal depth variable selection"16,30 using the variable select routine ${ }^{30}$ with parameter settings $n_{\text {tree }}=1000$, node size $=2$, and $\mathrm{n}_{\text {split }}=10$. The "minimal depth" of a variable is a statistic that can be used to measure the "predictiveness" of this variable. A particular advantage of minimal depth is that it allows for robust identification of predictive variables in high-dimensional data sets by thresholding. ${ }^{16}$ The covariates identified by this algorithm were then used to train final prognostic RSFs ( $\mathrm{n}_{\text {tree }}=1000$, node size $=3, \mathrm{n}_{\text {split }}=10$ ), with the objective to predict an individual risk for each patient. To obtain unbiased estimates for each patient, training and prediction were performed using 10 -fold cross validation, as implemented in the "mlr" framework. ${ }^{31}$

The median of the predicted individual risks was used to stratify patients into 2 evenly sized groups with low and high predicted risk; differences in overall survival between these groups were assessed by Kaplan-Meier analysis. A 2-sided log-rank test was used to test for a difference in overall survival between the 2 groups. Moreover, the association of predicted risk with overall survival was assessed with a univariate Cox proportional hazards models.

$P$ values of less than 0.05 were considered to indicate statistical significance.

\section{RESULTS}

\section{Patients}

Of the 71 patients screened for study entry, 66 patients fulfilled the inclusion criteria (mean age \pm standard deviation, $66 \pm 13$ years; age range, 26-79 years; 41 male; mean age \pm standard deviation, $60 \pm 13$ years; age range, $30-78$ years; 25 female; mean age \pm standard deviation, $58 \pm 12.6$ years; age range, $26-79$ years). For $n=5$ patients, no 3D CE MRI scans could be retrieved. $\mathrm{N}=32$ patients underwent microsurgical resection; $n=34$ patients underwent stereotactic biopsy. All patients were scheduled to receive the same treatment regime consisting of radiotherapy and concomitant and adjuvant temozolomide $(\mathrm{RCx} /$ TMZ), ${ }^{21} \mathrm{n}=64$ patients begun $\mathrm{RCx}$, and $\mathrm{n}=55$ patients completed RCx. Overall survival was measured from the date of initial MRI to the date of death (if applicable). At the time of last follow-up, 58 patients had died. Twelve patients had undergone imaging at $1.5 \mathrm{~T}, 54$ patients were imaged at $3 \mathrm{~T}$.

\section{Tumor Segmentation}

While the majority of patients $(n=62)$ had a single contrastenhancing tumor, 4 patients presented with more than 1 contrastenhancing lesion, which were not directly connected. Representative tumor segmentations on 3 T CE T1-weighted images are shown in Figure 1.

\section{Image Features}

Many of the 208 image features exhibit strong intrapatient correlations, which can be appreciated in a heat map of the feature correlation matrix, displayed in Figure 2. Large groups of features were found to exhibit strong positive or negative correlations, most notably pronounced for the several groups of pfTAS features, suggesting feature redundancy.

\section{Random Survival Forests}

From the 208 features derived from the segmented tumors, the minimal depth variable selection algorithm identified 20 features with high importance, displayed in Figure 3. The feature with the highest importance was pfTAS_136, closely followed by pfTAS_103. The RSF trained with these 20 features achieved mean concordance index of $67.7 \%$. The predicted individual risks ranged from $10.1 \%$ to $53.8 \%$, with a median risk of $38.7 \%$.

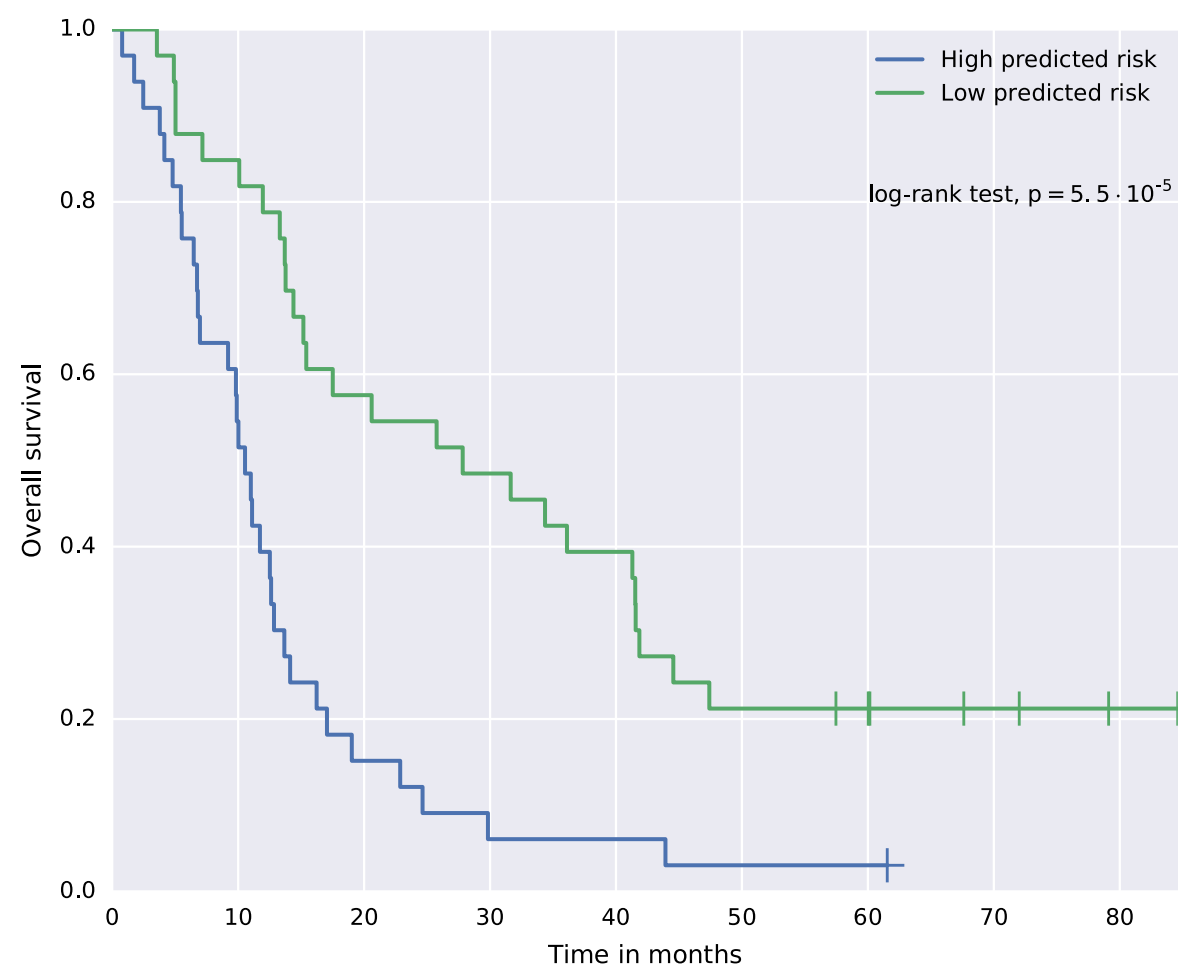

FIGURE 4. Association between predicted individual risk and overall survival. Patients were stratified in 2 equal-sized groups by the median of the predicted risk. The Kaplan-Meier plot shows clear separation of the groups with low (green) and high (blue) predicted risk. Figure 4 can be viewed online in color at www.investigativeradiology.com. 


\section{Association of Predicted Risk With Overall Survival}

The association between predicted individual risk with overall survival is illustrated by the Kaplan-Meier plot in Figure 4. Stratifying all patients into 2 groups with low (coded in green) and high (coded in blue) predicted risk, thresholded by the median value, reveals that patients with longer overall survival accumulate in the group with low predicted mortality. The survival curves of both groups are clearly separated. The difference, assessed with a 2-sided log-rank test, is highly significant $\left(P=5.5 \times 10^{-5}\right)$. Figure 5 provides additional insight into the relation between predicted risk and overall survival. Patients with short overall survival tend to accumulate in the top left of the plot. The median of predicted risk, indicated by the green line, separates this cluster from most of the long-term survivors.

The associations between overall survival and clinical variables, assessed with univariate Cox proportional hazard models, are displayed in Table 1. Briefly, young age, higher initial KPS, methylated $\mathrm{O}^{6}$ methylguanine DNA methyltransferase promoter status, and low predicted risk were identified as favorable prognostic covariates for overall survival in univariate Cox proportional hazard models, whereas patient sex and mode of surgery had no association with overall survival (Table 1).

\section{DISCUSSION}

In the present study, we analyzed CE T1-weighted MRI scans of 66 patients with newly diagnosed GBM with a radiomic approach. From each manually segmented contrast-enhancing lesion, 208 quantitative
TABLE 1. Prognostic Factors in Cox Proportional Hazards Models

\begin{tabular}{lccl}
\hline Variable & \multicolumn{3}{c}{$\mathbf{9 5 \% \text { Confidence }}$} \\
\hline Age $\dagger$ & Relative Risk & Interval & \multicolumn{1}{c}{$\boldsymbol{P}^{*}$} \\
Sex female & 1.03 & $1.01-1.05$ & $\mathbf{0 . 0 2 9}$ \\
KPS $\dagger$ & 1.36 & $0.80-2.3$ & 0.50 \\
MGMT promoter methylated & 0.96 & $0.94-0.99$ & $\mathbf{0 . 0 2 2}$ \\
Mode of surgery (resection) & 0.38 & $0.22-0.67$ & $\mathbf{0 . 0 0 4 4}$ \\
Predicted risk $\ddagger$ & 0.85 & $0.51-1.43$ & 0.54 \\
& 1.038 & $1.015-1.062$ & $\mathbf{0 . 0 0 5 9}$
\end{tabular}

${ }^{*} P$ values were adjusted for multiple testing using the Holm correction.

$\dagger$ Continuous scale, per 1 year increase.

†Continuous scale, per $1 \%$ increase; KPS, $10 \%$ increments.

Boldface indicates a significant difference $(P<0.05)$.

KPS indicates Karnofsky performance score; MGMT, $\mathrm{O}^{6}$-methylguanine DNA methyltransferase.

image features were derived, resulting in a high-dimensional parameter space. A variable selection algorithm identified a small number of prognostic features in this parameter space. These selected features were then used to train final RSFs, which predicted individual risk for each included subject. Stratifying the patients into 2 groups with high and low predicted mortality revealed a strongly significant difference

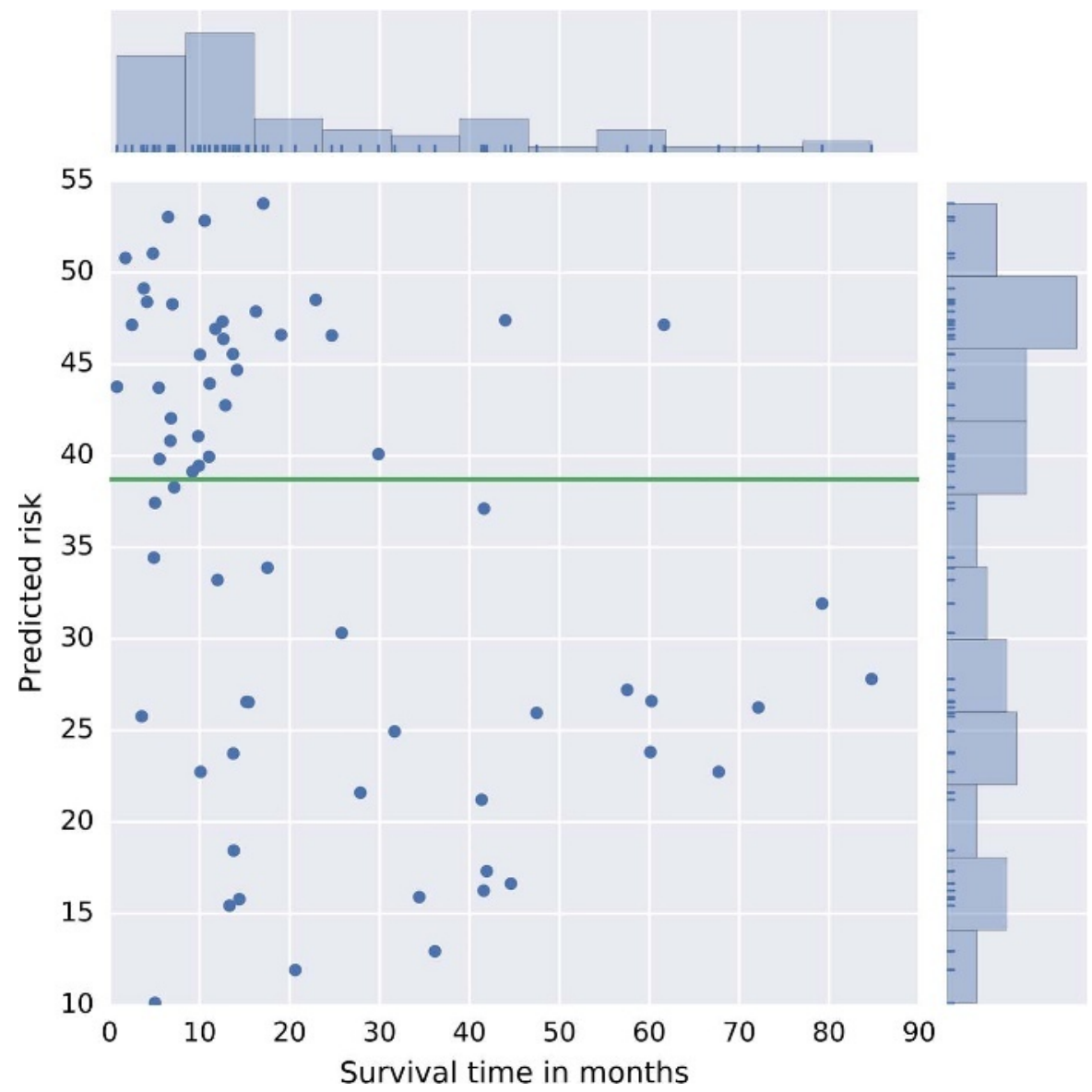

FIGURE 5. Association between predicted risk and overall survival. The green line indicates the median predicted risk that was used to stratify patient groups in Figure 4. The plot margins show the distribution of overall survival (top) and predicted risk (right) in the patient sample. Figure 5 can be viewed online in color at www.investigativeradiology.com. 
in overall survival and a proportional hazards model revealed a highly significant association of the predicted risk with overall survival.

The set of important variables, as selected by minimal-depth variable selection, contained mainly texture parameters from the pfTAS and Haralick groups. In addition, 3 features characterizing tumor shape and 1 feature from the first-order signal intensity groups were included in the final set. The final model set did not contain any features characterizing tumor size, but strong positive correlations between size-related features and distinct texture features (eg, pfTAS136) indicate that information about the contrast-enhancing tumor shape is implicitly included in the final prognostic model.

In a recent study, ${ }^{13}$ a radiomic analysis was used to predict 2-year survival from pretreatment computed tomography images in patients with lung cancer. In this study, the survival analysis was cast as a classification task and random forests, among other machine learning classifiers, were used to predict whether the patient survived longer than 2 years. A similar classification approach was also used to predict progression-free survival in nasopharyngeal carcinoma from T1-weighted MRI data. ${ }^{32}$ While this is a valid strategy, the classification approach relies on the definition of an arbitrary threshold value. Furthermore, the binary prediction whether patients survive longer than the set threshold is of limited value in a clinical setting. In our study, we overcame these limitations by training RSFs on the entire parameter space, with the purpose to develop a prognostic model that does not rely on arbitrary thresholds and that predicts risk as a continuous variable.

Random survival forests are a recent extension ${ }^{17}$ of the popular random forest method and have been successfully used in the analysis of genomic microarray data. ${ }^{18}$ This is a structurally similar problem where sample sizes are limited and the large number of parameters can exceed the number of samples, sometimes by far. ${ }^{18}$ Moreover, many of the genomic features may be highly correlated, whereas others may have no association at all with the outcome variable. These properties are also characteristic for many radiomics data sets. A feature selection step before further analysis ${ }^{13}$ is often used to reduce the dimensionality of the parameter space. In the present study, we have selected important features by means of training an initial RSF on the entire data and assessing the importance of variables by their minimal depth. ${ }^{16}$

Recently, Kickingereder et $\mathrm{al}^{33}$ have used a radiomic analysis to predict survival of GBM patients from baseline MRI, including fluidattenuated inversion recovery as well as precontrast and postcontrast T1 images. In their study, a feature selection step based on supervised principal components was followed by proportional hazards regression. Random survival forests, as used in our study, have the advantage to intrinsically identify important variables and, as a nonparametric method, do not rely on a restrictive proportional hazards assumption. Important features selected in Kickingereder et $\mathrm{al}^{33}$ were exclusively based on fluid-attenuated inversion recovery images. Therefore, a further increase in prognostic value of the presented approach can be expected through inclusion of additional MRI scan contrasts or even imaging modalities. Additional image contrast would also allow the use of automated tumor segmentation, ${ }^{34,35}$ thereby increasing scalability, reproducibility, and hence clinical usefulness.

A limitation of the present study is the utilization of CE 3D T1weighted images only. Nevertheless, our analysis revealed a highly prognostic value of this single MRI scan contrast alone. In this study, tumors were contoured manually, which is time-consuming and user dependent. This highlights the need for automated tumor segmentation, which would minimize user bias and enable larger-scale studies. In addition, we have focused the feature selection on the prognostic value of the features. An important criterion for potential clinical application is the stability of features with respect to volume definition, MR scanner, or sequence settings, ${ }^{36}$ which we have not investigated. Due to the small sample size of our study, we were not able to split our data into training and test data sets. To circumvent this issue, predicted risk was calculated using 10-fold cross validation, thus ensuring an unbiased prediction within this sample. ${ }^{37}$ Still, further studies and validation in independent data sets are required to ensure that our approach generalizes to independent data. Moreover, we have not tuned the hyperparameters of our random forests, but have used the default settings of the $\mathrm{R}$ package to demonstrate that a radiomics approach can extract prognostic information from baseline MRI. Presumably, the prognostic performance can be increased considerably, when hyperparameters are optimized in further studies with larger sample sizes.

In conclusion, this study indicates that baseline CE T1-weighted MRI in GBM patients contains prognostic information, which can be accessed and used to build prognostic models by means of an ensemble machine learning technique, RSFs. In the future, this approach can be readily extended to include additional MR contrasts, functional imaging, or even additional modalities such as CT or PET.

\section{REFERENCES}

1. Louis DN, Ohgaki H, Wiestler OD, et al. The 2007 WHO Classification of Tumours of the Central Nervous System. Acta Neuropathol. 2007;114:97-109.

2. Gutman DA, Cooper LA, Hwang SN, et al. MR imaging predictors of molecular profile and survival: multi-institutional study of the TCGA glioblastoma data set. Radiology. 2013;267:560-569.

3. Hegi ME, Diserens AC, Gorlia T, et al. MGMT gene silencing and benefit from temozolomide in glioblastoma. $N$ Engl J Med. 2005;352:997-1003.

4. Quant EC, Wen PY. Novel medical therapeutics in glioblastomas, including targeted molecular therapies, current and future clinical trials. Neuroimaging Clin NAm. 2010;20:425-448.

5. Koshy M, Villano JL, Dolecek TA, et al. Improved survival time trends for glioblastoma using the SEER 17 population-based registries. J Neurooncol. 2012; 107:207-212.

6. Stupp R, Hegi ME, van den Bent MJ, et al. Changing paradigms - an update on the multidisciplinary management of malignant glioma. Oncologist. 2006;11: 165-180.

7. Aerts HJWL, Velazquez ER, Leijenaar RTH, et al. Decoding tumour phenotype by noninvasive imaging using a quantitative radiomics approach. Nat Commun. 2014;5. Available at: http://www.nature.com/doifinder/10.1038/ncomms5006. Accessed February 4, 2015.

8. Lambin P, van Stiphout RG, Starmans MH, et al. Predicting outcomes in radiation oncology —multifactorial decision support systems. Nat Rev Clin Oncol. 2013;10: 27-40.

9. Lambin P, Rios-Velazquez E, Leijenaar R, et al. Radiomics: extracting more information from medical images using advanced feature analysis. Eur J Cancer. 2012; 48:441-446.

10. Gillies RJ, Kinahan PE, Hricak H. Radiomics: images are more than pictures, they are data. Radiology. 2016;278:563-577.

11. Wibmer A, Hricak H, Gondo T, et al. Haralick texture analysis of prostate MRI: utility for differentiating non-cancerous prostate from prostate cancer and differentiating prostate cancers with different Gleason scores. Eur Radiol. 2015;25 2840-2850.

12. Agner SC, Rosen MA, Englander S, et al. Computerized image analysis for identifying triple-negative breast cancers and differentiating them from other molecular subtypes of breast cancer on dynamic contrast-enhanced MR images: a feasibility study. Radiology. 2014;272:91-99.

13. Parmar C, Grossmann P, Bussink J, et al. Machine learning methods for quantitative radiomic biomarkers. Sci Rep. 2015;5:13087.

14. Coroller TP, Grossmann P, Hou Y, et al. CT-based radiomic signature predicts distant metastasis in lung adenocarcinoma. Radiother Oncol. 2015;114:345-350.

15. Breiman L. Random forests. Mach Learn. 2001;45:5-32.

16. Ishwaran H, Kogalur UB, Chen X, et al. Random survival forests for highdimensional data. Stat Anal Data Min. 2011;4:115-132.

17. Ishwaran H, Kogalur UB, Blackstone $\mathrm{EH}$, et al. Random survival forests Ann Appl Stat. 2008;2:841-860.

18. Chen X, Ishwaran H. Random forests for genomic data analysis. Genomics. 2012 99:323-329.

19. Hsich E, Gorodeski EZ, Blackstone EH, et al. Identifying important risk factors for survival in patient with systolic heart failure using random survival forests. Circ Cardiovasc Qual Outcomes. 2011;4:39-45.

20. Villanueva A, Hoshida Y, Battiston C, et al. Combining clinical, pathology, and gene expression data to predict recurrence of hepatocellular carcinoma. Gastroenterology. 2011;140:1501-1512.e2 
21. Suchorska B, Jansen NL, Linn J, et al. Biological tumor volume in ${ }^{18}$ FET-PET before radiochemotherapy correlates with survival in GBM. Neurology. 2015;84: 710-719.

22. Wolf I, Vetter M, Wegner I, et al. The Medical Imaging Interaction Toolkit. Med Image Anal. 2005;9:594-604.

23. Haralick RM, Shanmugam K, Dinstein IH. Textural features for image classification. Syst Man Cybern IEEE Trans On. 1973;6:610-621.

24. Coelho LP. Mahotas: Open source software for scriptable computer vision. J Open Res Softw. 2013;1. Available at: http://openresearchsoftware.metajnl.com/articles/ 10.5334/jors.ac/. Accessed June 14, 2016.

25. Hamilton NA, Pantelic RS, Hanson K, et al. Fast automated cell phenotype image classification. BMC Bioinformatics. 2007;8:110.

26. Coelho LP, Ahmed A, Arnold A, et al. Structured literature image finder: extracting information from text and images in biomedical literature. In: Blaschke $\mathrm{C}$, Shatkay H, eds. Linking Literature, Information, and Knowledge for Biology. Lecture Notes in Computer Science. Berlin, Germany: Springer; 2010:23-32. Available at: http://link.springer.com/chapter/10.1007/978-3-642-13131-8_4. Accessed June 14, 2016

27. Gorgolewski K, Burns CD, Madison C, et al. Nipype: a flexible, lightweight and extensible neuroimaging data processing framework in python. Front Neuroinform. 2011;5:13.

28. R Core Team. R: A Language and Environment for Statistical Computing. Vienna, Austria: R Foundation for Statistical Computing; 2014. Available at: http://www. R-project.org. Accessed August 5, 2016.
29. Holm S. A simple sequentially rejective multiple test procedure. Scand J Stat. 1979;6:65-70.

30. Ishwaran H, Kogalur UB. Random Forests for Survival, Regression and Classification $(R F-S R C)$. manual; 2016. Available at: https://cran.r-project.org/package= randomForestSRC. Accessed August 5, 2016.

31. Bischl B, Lang M, Kotthoff L, et al. mlr: Machine Learning in R. J Mach Learn Res. 2016;17:1-5.

32. Farhidzadeh H, Kim JY, Scott JG, et al. Classification of progression free survival with nasopharyngeal carcinoma tumors. In: Vol 9785.; 2016:97851I-97851I-7. Available at: http://dx.doi.org/10.1117/12.2216976. Accessed June 16, 2016.

33. Kickingereder P, Burth S, Wick A, et al. Radiomic profiling of glioblastoma: identifying an imaging predictor of patient survival with improved performance over established clinical and radiologic risk models. Radiology. 2016;280:880-889.

34. Menze BH, Jakab A, Bauer S, et al. The multimodal brain tumor image segmentation benchmark (BRATS). IEEE Trans Med Imaging. 2015;34:1993-2024.

35. Goetz M, Weber C, Binczyk F, et al. DALSA: domain adaptation for supervised learning from sparsely annotated MR images. IEEE Trans Med Imaging. 2016; 35:184-196.

36. Mackin D, Fave X, Zhang L, et al. Measuring computed tomography scanner variability of radiomics features. Invest Radiol. 2015;50:757-765.

37. James G, Witten D, Hastie T, et al. An Introduction to Statistical Learning. Springer New York: New York, NY; 2013. Available at: http://link.springer.com/ 10.1007/978-1-4614-7138-7. Accessed July 27, 2016. 